\title{
Water-soluble octaacid capsule as a reaction container: Templated photodimerization of indene in water
}

\author{
Anand Parthasarathy* and V. Ramamurthy* \\ Department of Chemistry, University of Miami, Coral Gables, FL 33146 \\ aparthas.ufl@gmail.com \\ murthy1@miami.edu
}

\begin{abstract}
In the context of green chemistry, identifying strategies to carry out organic reactions in aqueous medium in the absence of organic and inorganic reagents is important. We show below that octa acid (OA), a water-soluble cavitand, is capable of solubilizing water insoluble reactant in water and yielding selective product by confining the reactants and intermediates during a light initiated reaction. By employing indene as the substrate we have observed that the OA capsule upon irradiation facilitates the formation of anti-head-tail dimer, the isomer that is generally not obtained upon direct excitation and triplet and electron transfer sensitizations in isotropic organic solvents.
\end{abstract}




\section{Introduction}

Conducting photochemical reactions within confined spaces has been an important strategy to control the reactivity of reactants and intermediates and channelize photoreactions to obtain high product selectivity.[1] For this purpose, various media such as zeolites, clays, crystals, cyclodextrins, cucurbiturils, calixarenes, micelles, dendrimers, polymers, host-guest complexes and natural proteins have been successfully explored during the last few decades.[217] These studies have clearly shown that the confinement and weak interactions provided by a host can steer reactivity of guest molecules resulting in highly selective reactions that are otherwise challenging in isotropic media. Reactions of a guest molecule in confined spaces could be visualized to occur within a 'reaction cavity' with a defined boundary and some amount of 'free space' surrounding the reactant within the cavity.[18] The extent of selectivity within such a reaction cavity depends on this free space, flexibility (hard or soft) of the boundary and weak interactions that hold the guest within the host. It is important to note that confined hosts offer an environment that is more free than solids but more restricted than isotropic solutions.

Herein, we report photo-dimerization of indene molecules held together within the inner cavity of a water-soluble host, octa acid (indene ${ }_{2} @ \mathrm{OA}_{2}$, Scheme 1). Cavitand OA is known to self-assemble into a dimeric capsule in presence of appropriate hydrophobic guest molecules.[11, $19,20]$ In spite of being water soluble, polarity of OA capsules' interior resembles that of organic solvents. Thus guest encapsulation by OA makes it possible to conduct organic reactions in aqueous medium yet in completely non-polar organic solvent-like microenvironment.[21-23] Results of our studies presented here further strengthen our postulate that free space around the guest molecule along with weak non-covalent guest-host interactions can significantly influence the outcome of photodimerization of encapsulated olefin molecules. Indene molecules 
encapsulated within OA capsule upon photoexcitation yields anti-head-tail dimer, the dimer that has not been obtained through conventional photochemical techniques such as direct excitation and energy and electron transfer sensitizations. The results described below bring out the uniqueness of the OA capsule as a reaction container.

\section{Experimental}

Indene was procured from Aldrich and used as such. All ${ }^{1} \mathrm{HNMR}$ spectra were recorded using a Bruker $500 \mathrm{MHz} \mathrm{NMR}$ at $27^{\circ} \mathrm{C}$. OA was synthesized as per the reported procedure and was dissolved in sodium borate buffer. The borate buffer OA solution was used for NMR and photochemical experiments.

\section{Protocol for Complexation of indene with $O A$}

Six hundred $\mu \mathrm{L}$ of a $\mathrm{D}_{2} \mathrm{O}$ stock solution of host $\mathbf{O A}(1 \mathrm{mM} / 10 \mathrm{mM}$ sodium tetraborate) was taken in a NMR tube. Calculated amount of indene stock solution $(60 \mathrm{mM}$ solution in DMSO- $d_{6}$ ) was gradually added following which the NMR tube was shaken rigorously to achieve complexation (typically 0.25 equivalents of guest was added during each addition).The NMR spectrum was recorded after ca. 5 minutes. The titration was continued until the addition of excess indene did not alter the guest@ host NMR spectrum. In general, the addition of excess guest led to turbid solutions. NMR spectrum was also recorded after 24 h to test the stability of the complex and no change was observed. Spectra were recorded at room temperature under aerated conditions on Bruker $500 \mathrm{MHz} \mathrm{NMR}$ at $25^{\circ} \mathrm{C}$. 
Irradiation of indene included within $O A$ capsule and isolation and analysis of the photoproducts:

A 1:1 guest to host complex was made by adding a DMSO- $d_{6}$ stock solution of indene $\left(10 \mu \mathrm{l}\right.$ of $300 \mathrm{mM}$ solution in DMSO- $\left.d_{6}\right)$ to OA in a NMR tube $(0.6 \mathrm{~mL}, 5 \mathrm{mM}$ OA in $50 \mathrm{mM}$ sodium tetraborate buffer in $\mathrm{D}_{2} \mathrm{O}$ ). The resulting solution was shaken and sonicated to achieve complexation. The clear solution was purged with dry $\mathrm{N}_{2}$ for $20 \mathrm{~min}$ and irradiated using a 450 W medium pressure Hg lamp for different time periods, and the reaction was followed by NMR. After photolysis, the products were extracted from the aqueous host solution with $\mathrm{CDCl}_{3}$, dried over anhydrous $\mathrm{Na}_{2} \mathrm{SO}_{4}$ and analyzed on an HP-5890 series II gas chromatograph fitted with an SE-30 capillary column. Subsequently, products were also identified by NMR and GC-MS.

\section{Results and Discussion}

The structures of host OA and guest indene are shown in Scheme 1. OA, which is sparingly soluble in water at neutral $\mathrm{pH}$, is more soluble $\left(\sim 10^{-2} \mathrm{M}\right)$ in a slightly basic aqueous solution ( $\mathrm{pH} \sim 9)$. At such concentrations two molecules of OA self-assemble in the presence of a suitable guest molecule(s) to form 1:2 or 2:2 guest to host complex that are denoted as guest@ $\mathrm{OA}_{2}$ or guest $2_{2}(\mathrm{OA})_{2}$ and they both are generally called as capsules. In presence of indene association of two molecules of OA results in a closed capsule within which the dimerization of indene proceeds. 
(a)

\section{Host}

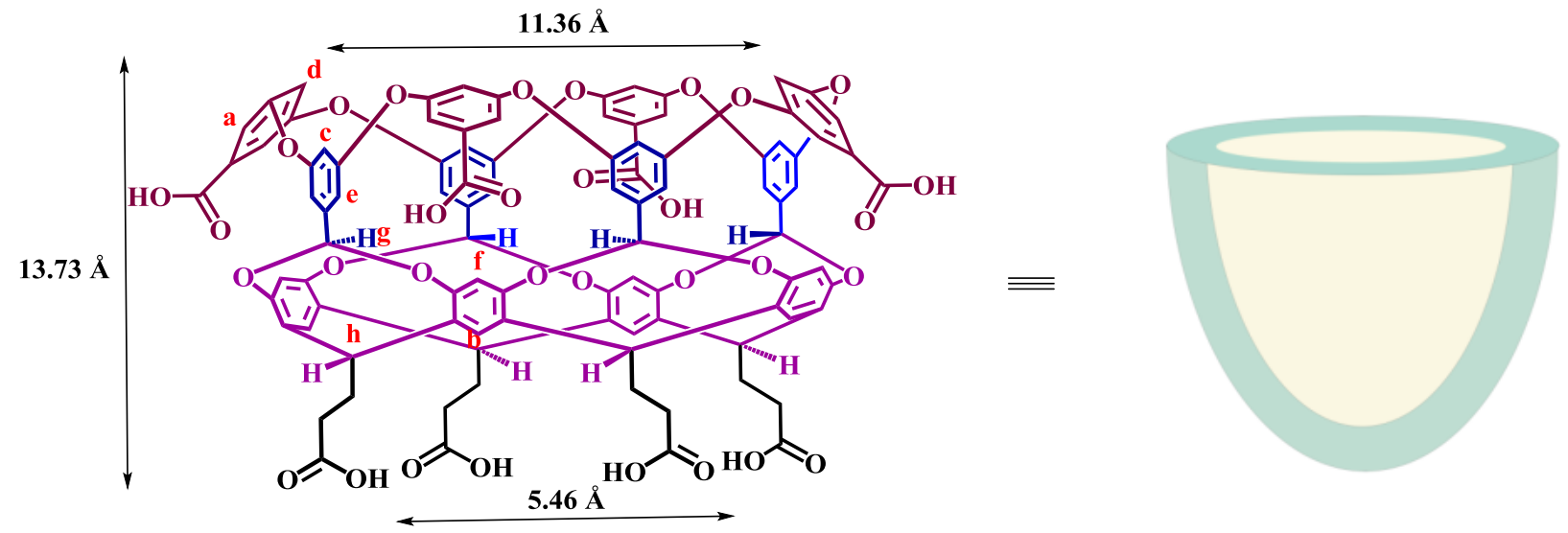

(b)

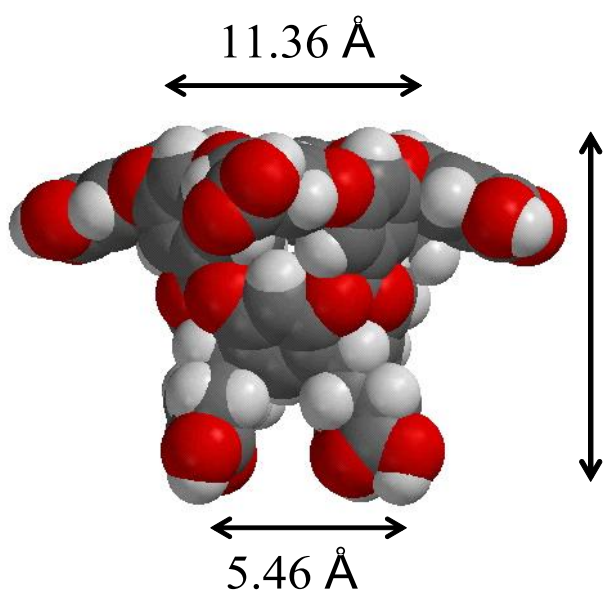

$13.73 \AA$

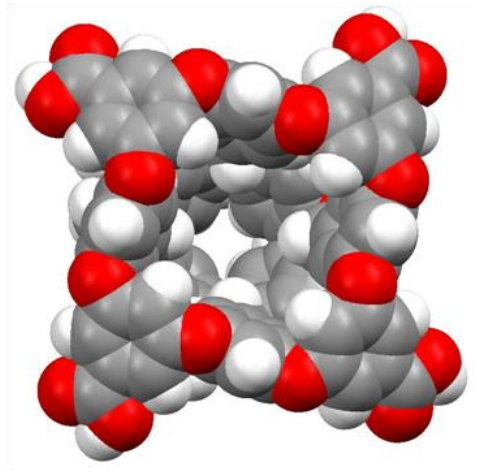

(c)

Guest<smiles>C1=Cc2ccccc2C1</smiles>

Indene

Scheme 1. (a) Structure and cartoon representation of the host octa acid (OA), (b) CPK models showing the dimensions (left) and OA reaction cavity from top (right), and (c) structure of the guest indene

We inferred the formation of 2:2 complex of indene with OA by ${ }^{1} \mathrm{H}$ NMR titration experiments and 2-D NMR spectra (Figures 1-3). As can be seen in Figure 1, spectra for 1:2 and $2: 2$ ratios of indene to $\mathrm{OA}$ are different and addition of more than $2: 2$ equivalent of indene did 
not further change the spectra. This suggested that OA forms a stable 2:2 complex with indene. Further, the fact that the aromatic host signals are realigned but not split when the guest:host stoichiometry is 2:2 suggested the guests are placed in a symmetric fashion within the OA capsule.

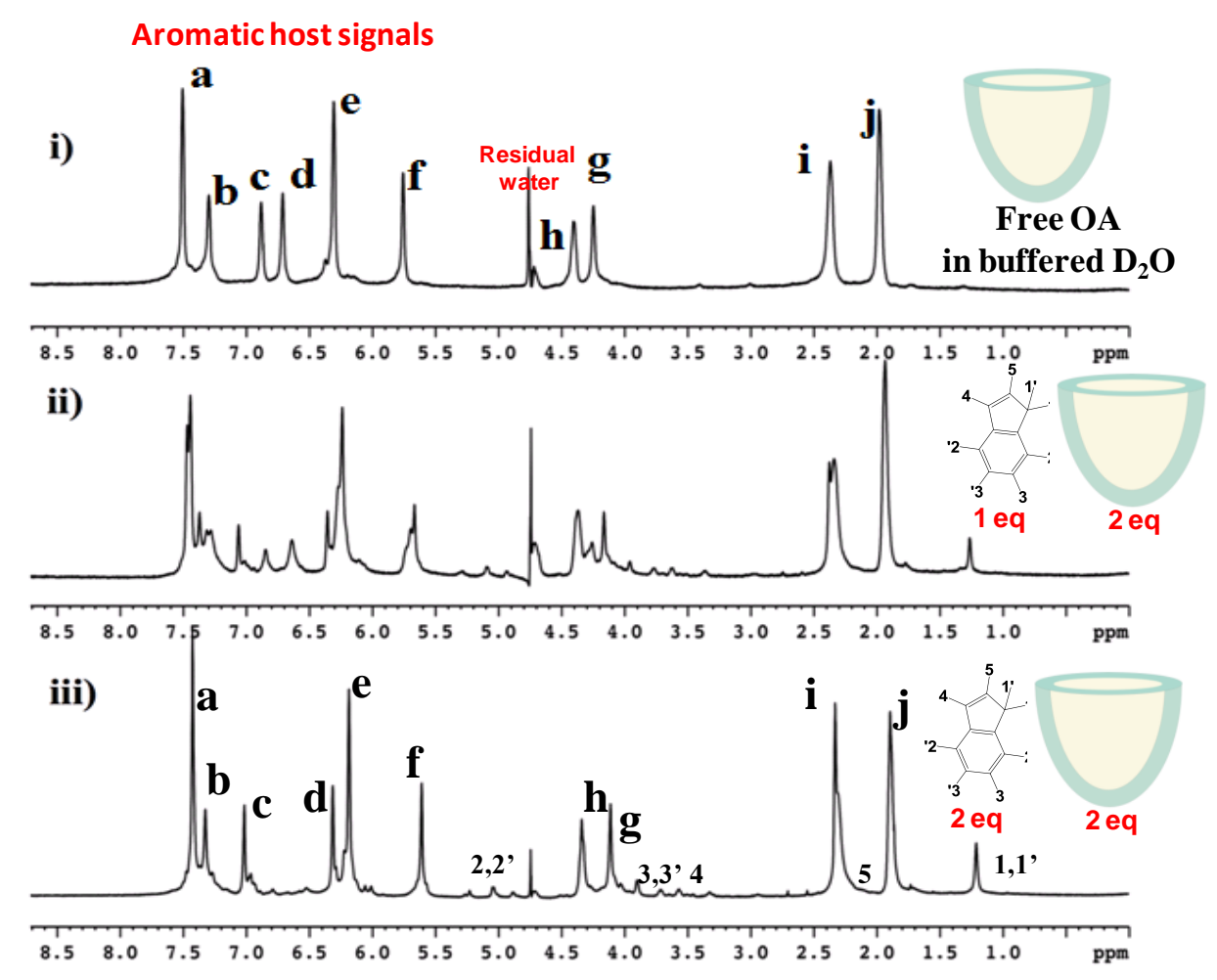

Figure 1. ${ }^{1} \mathrm{H}$ NMR showing the formation of $2: 2$ complex between indene and OA $(1 \mathrm{mM})$ in buffered $\left.\mathrm{D}_{2} \mathrm{O}(500 \mathrm{MHz}) . \mathrm{i}\right)$ Free OA. ii) upon addition of 0.5 equivalents of indene to 1 equivalent of $\mathrm{OA}$, and (iii)upon addition of 1 equivalent of indene to 1 equivalent of OA (see Scheme 1 for labeling of OA proton signals).

COSY and NOESY experiments were carried out to further identify the guest signals and gain insight into the orientation of guest molecules within the interior of the capsular complex (Figures $\mathbf{2}$ and 3). The methylene proton signals of indene showed NOE correlation with ' $\mathbf{H}_{\mathbf{d}}$ ' signal of host, which is present at the 3rd row (top) of OA (Scheme 1). The aromatic protons 

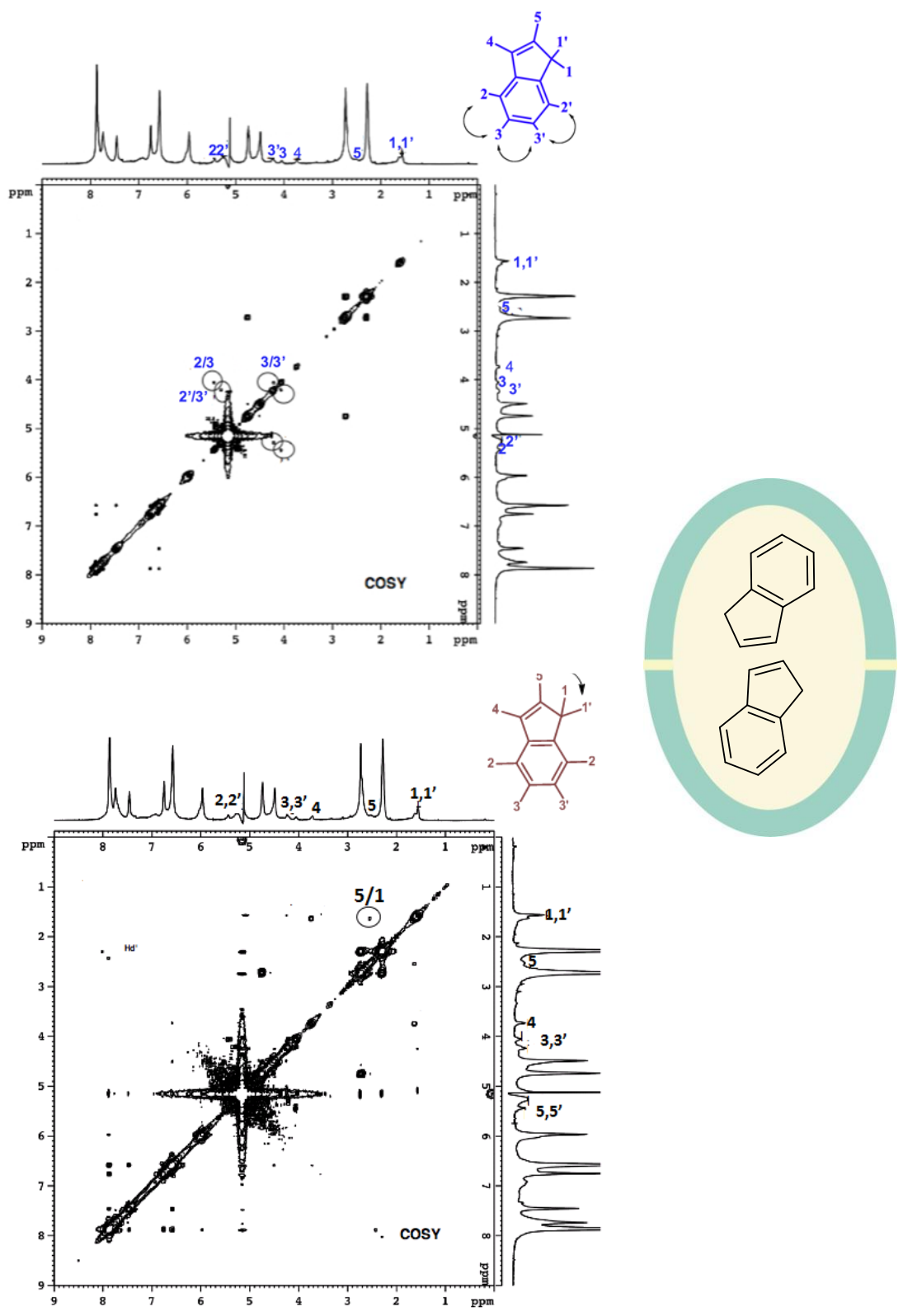

Figure 2. COSY spectra (top and bottom) of (indene $)_{2} @(\mathrm{OA})_{2}(500 \mathrm{MHz}$, [indene $]=[\mathrm{OA}]=5 \mathrm{mM}$ in $5 \times 10^{-3} \mathrm{M}$ buffered $\mathrm{D}_{2} \mathrm{O}$ ). Important correlations are chosen and marked for clarity. 

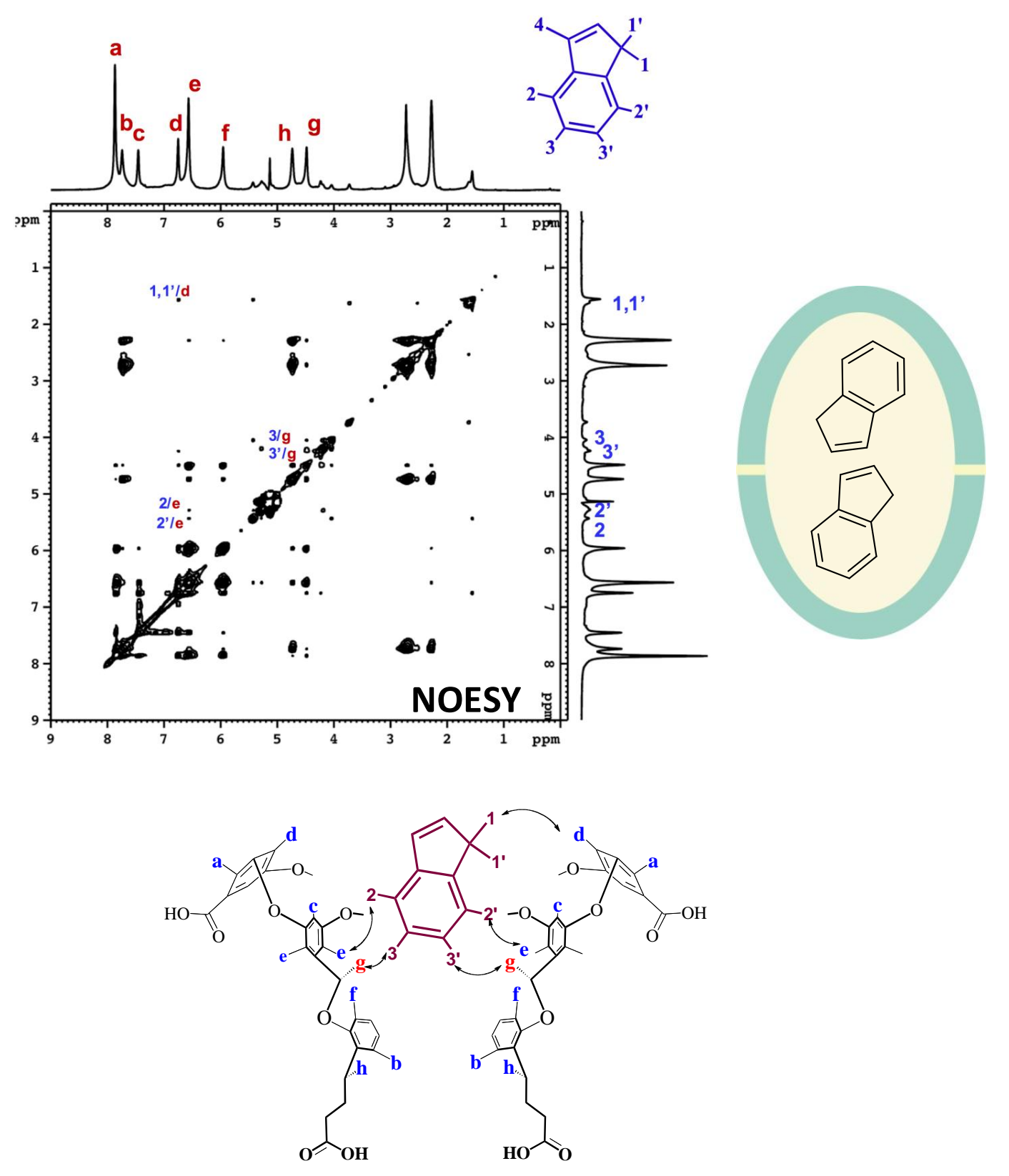

Figure 3. (top) NOESY spectrum of (indene $)_{2} @(\mathrm{OA})_{2}([\mathrm{OA}]=5 \mathrm{mM})$ in buffered $\mathrm{D}_{2} \mathrm{O}\left(5 \times 10^{-2}\right.$ $\mathrm{M}, 500 \mathrm{MHz})$ showing interactions of indene signals with host OA signals $(5 \mathrm{mM})$ in buffered $\mathrm{D}_{2} \mathrm{O}\left(5 \times 10^{-2} \mathrm{M}\right)$. (bottom) Pictorial representation of NOE correlations (one half of the capsule is shown for clarity).

of indene marked as 2,2' and 3,3' exhibited NOE correlations with ' $\mathbf{H}_{\mathbf{g}}$ ' and ' $\mathbf{H}_{\mathbf{e}}$ ' of OA (Figure 3,top). Based on these correlations we conclude that the guest is oriented in such a way that aromatic part of it is located at the narrower end of the capsule whereas the olefinic part occupies 
the middle wider region of the capsule. The proposed orientation is illustrated in (Figure 3, bottom).

\section{Photochemical results and discussion}

In isotropic media upon excitation indene yields dimers and polymers. The nature of the dimer formed depends on the polarity of the medium and the mode of activation.[24-28] Irradiation of indene in acetonitrile yields syn-head-head (1) as a major product (Scheme 2 and Table 1). Triplet sensitization with 4-methyoxyacetophenone gives about $84 \%$ of anti-head-head (2) (Scheme 2 and Table 1). [28]

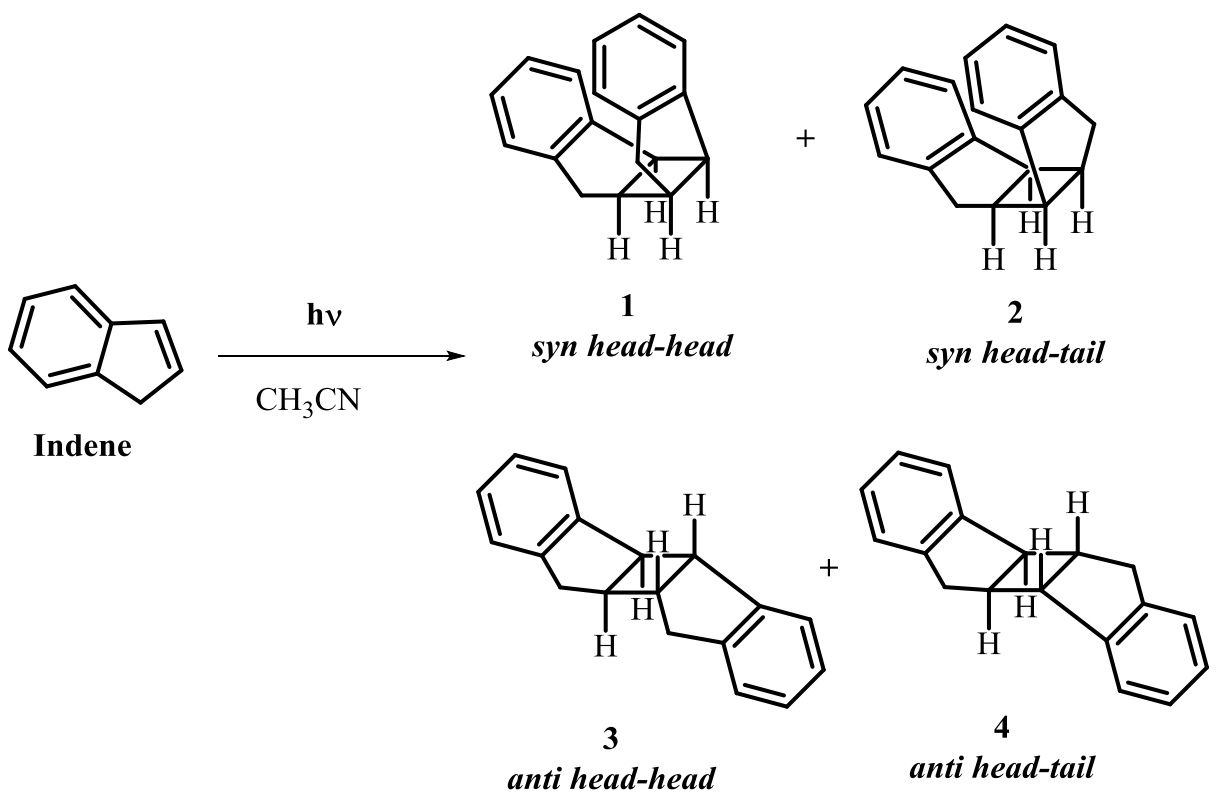

Scheme 2. Photodimerization of indene

Since indene forms 2:2 complex with OA, we were interested in examining how the confinement influences the reactivity of the encapsulated guest molecules. The 2:2 complex of (indene $)_{2} @(\mathrm{OA})_{2}\left([\mathrm{OA}]=2 \times 10^{-3} \mathrm{M}\right.$, [indene $\left.]=2 \times 10^{-3} \mathrm{M}\right)$ was irradiated in a Pyrex test tube with a medium pressure $\mathrm{Hg}$ vapor lamp. Comparison of aromatic host region of ${ }^{1} \mathrm{H}$ NMR spectra 
before and after irradiation of (indene $)_{2} @(\mathrm{OA})_{2}$ (Figure 4) indicated that chemical transformation of indene had taken place within the capsular complex (2-4 h of irradiation results in $>90 \%$ conversion of indene to products). After the photolysis, products were extracted with $\mathrm{CDCl}_{3}$ and analyzed by ${ }^{1} \mathrm{H}$ NMR (Figure 5) and GC-MS (Figure 6). The analysis of the two data suggested the major dimer to be anti-head-tail and the minor one to be anti-head-head.[25] About $90 \%$ of the starting material was converted to product in $5 \mathrm{~h}$ of irradiation. As a control experiment, indene $\left([\right.$ indene $\left.]=2 \times 10^{-3} \mathrm{M}\right)$ was irradiated in buffered water $\left(1 \times 10^{-2} \mathrm{M}\right)$ which is the medium used for the dissolution of the host, OA. This resulted in formation of anti-headhead as major dimer along with minor amounts of other dimers (Table 1) and the reaction was slow ( $c a 20 \mathrm{~h}$ of irradiation was required for $>90 \%$ conversion).
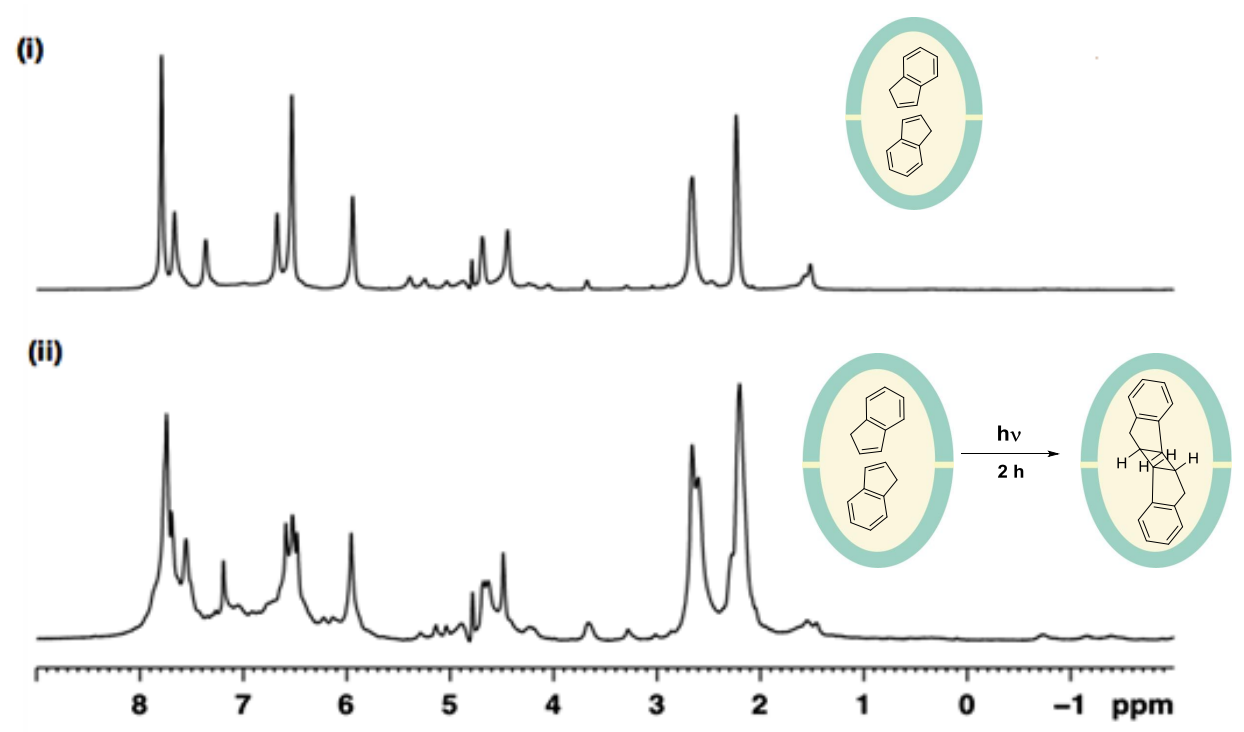

Figure 4. ${ }^{1} \mathrm{H}$ NMR showing of 2:2 complex between indene and $\mathrm{OA}(1 \mathrm{mM})$ in buffered $\mathrm{D}_{2} \mathrm{O}$ (500 MHz). (i) before photolysis, and (ii) after photolysis for $2 \mathrm{~h}$. 
Table 1. Photodimerization of indene under different conditions

\begin{tabular}{|l|l|l|l|l|}
\hline \multirow{2}{*}{ Medium } & \multicolumn{4}{c|}{ Product Distribution } \\
\cline { 2 - 5 } & $\begin{array}{c}\text { syn head- } \\
\text { head (1) }\end{array}$ & $\begin{array}{c}\text { syn head- } \\
\text { tail (2) }\end{array}$ & $\begin{array}{c}\text { anti head- } \\
\text { head (3) }\end{array}$ & $\begin{array}{c}\text { anti head- } \\
\text { tail (4) }\end{array}$ \\
\hline Buffer & 6.7 & 18.7 & 65.8 & 8.9 \\
\hline Buffer/acetophenone & 5.6 & 6.9 & 80.7 & 7.0 \\
\hline $\mathrm{OA} /$ indene (2:2) & 1.5 & 3.3 & 5.5 & 89.7 \\
\hline $\mathrm{CH}_{3} \mathrm{CN}^{\mathrm{a}}$ & 74 & 5 & 12 & 6 \\
\hline $\mathrm{CH}_{3} \mathrm{CN} / p$-methoxyacetophenone ${ }^{\mathrm{a}}$ & 3 & 5 & 84 & 8 \\
\hline $\mathrm{CH}_{3} \mathrm{CN} / \mathrm{N}$-methylacridinium iodide ${ }^{\mathrm{a}}$ & - & - & 96 & 4 \\
\hline
\end{tabular}

${ }^{a}$ L. Brancaleon et al., J. Am. Chem. Soc., 1998, 120, 4926-4933; ${ }^{\text {t }}$ The estimated yield is the average of 3 runs
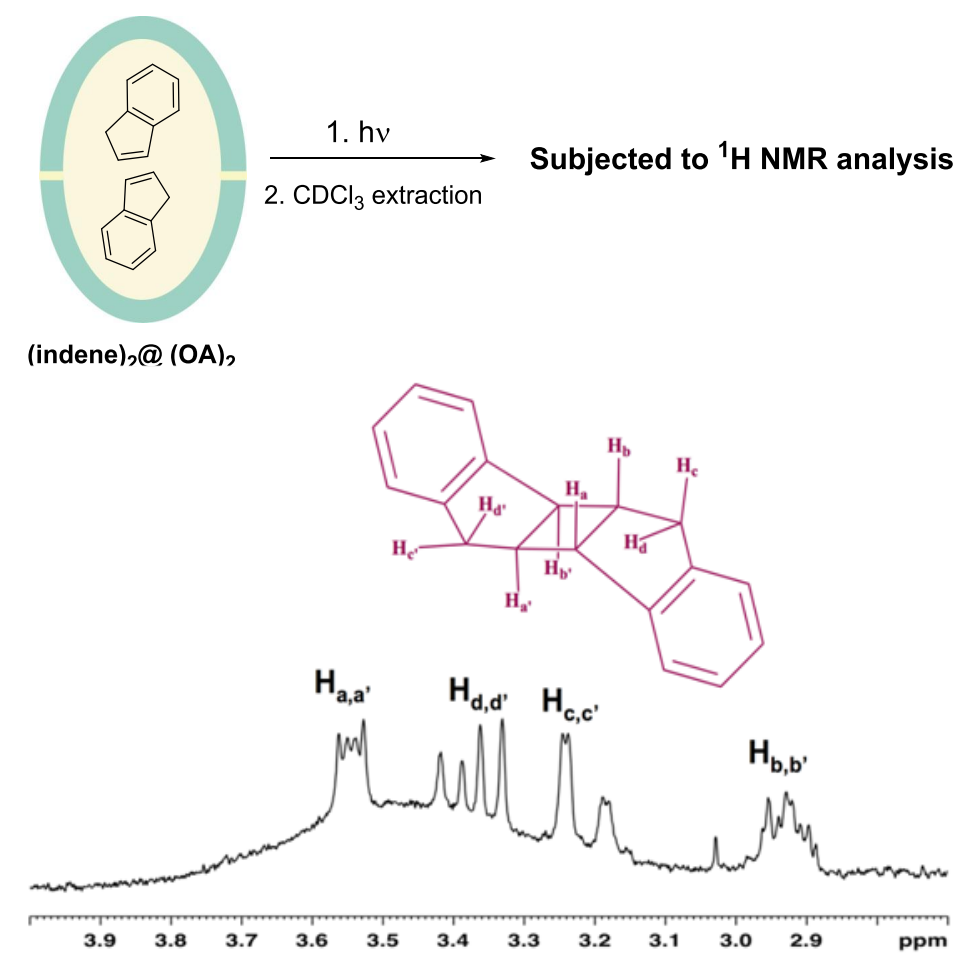

Figure 5. Photodimer obtained upon photolysis (indene) $)_{2} @(\mathrm{OA})_{2}$ in buffered $\mathrm{D}_{2} \mathrm{O}$ and extraction with $\mathrm{CDCl}_{3}$ (Assignment was made by comparing the spectrum with reported ${ }^{1} \mathrm{H}$ NMR of dimer). 


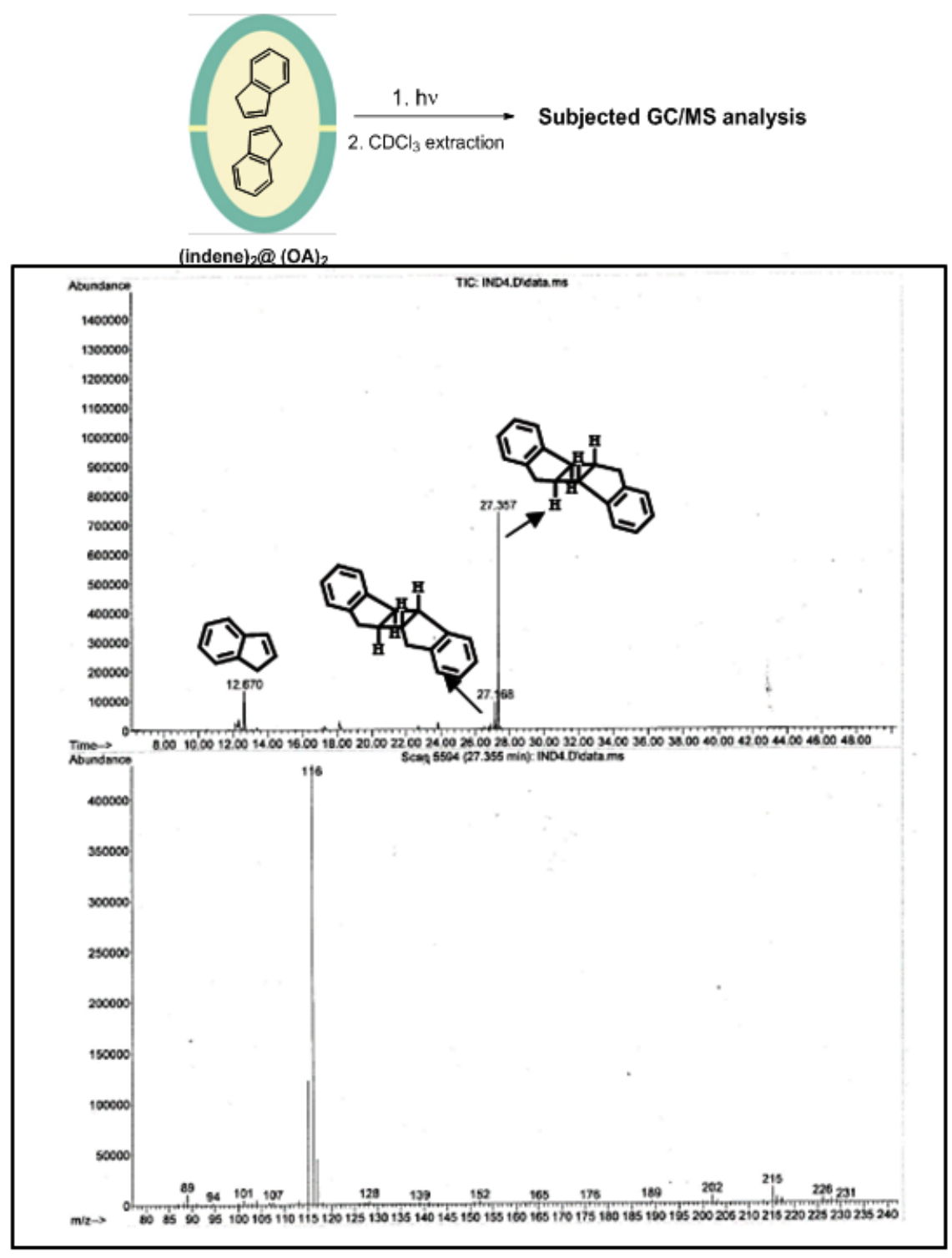

Figure 6. GC-MS trace of photodimer obtained upon photolysis indene@ $(\mathrm{OA})_{2}$ in buffered $\mathrm{D}_{2} \mathrm{O}$ and extraction with $\mathrm{CDCl}_{3}$.

Studies from our group have shown that OA capsule can control the reactivity of the included guest molecules.[19, 21, 22] Strikingly, indene photodimerization within OA capsule results in formation anti-head-tail dimer (>90\%) as the major isomer. What is more appealing is that this dimer is uncommon in organic solvents by both direct excitation (syn-head-head major product, 
Table 1) or by sensitization (anti-head-head major product by triplet and electron transfer sensitization, Table 1). Photodimerization of indene within NaY zeolites both by direct excitation and by using electron transfer sensitizers results in formation of syn-head-head and anti-headhead as major products within $\mathrm{NaX}$ zeolites.[28] The above results clearly reveal that OA capsule's inner phase is unique in steering the indene photodimerization towards the formation of anti-head-tail dimer.
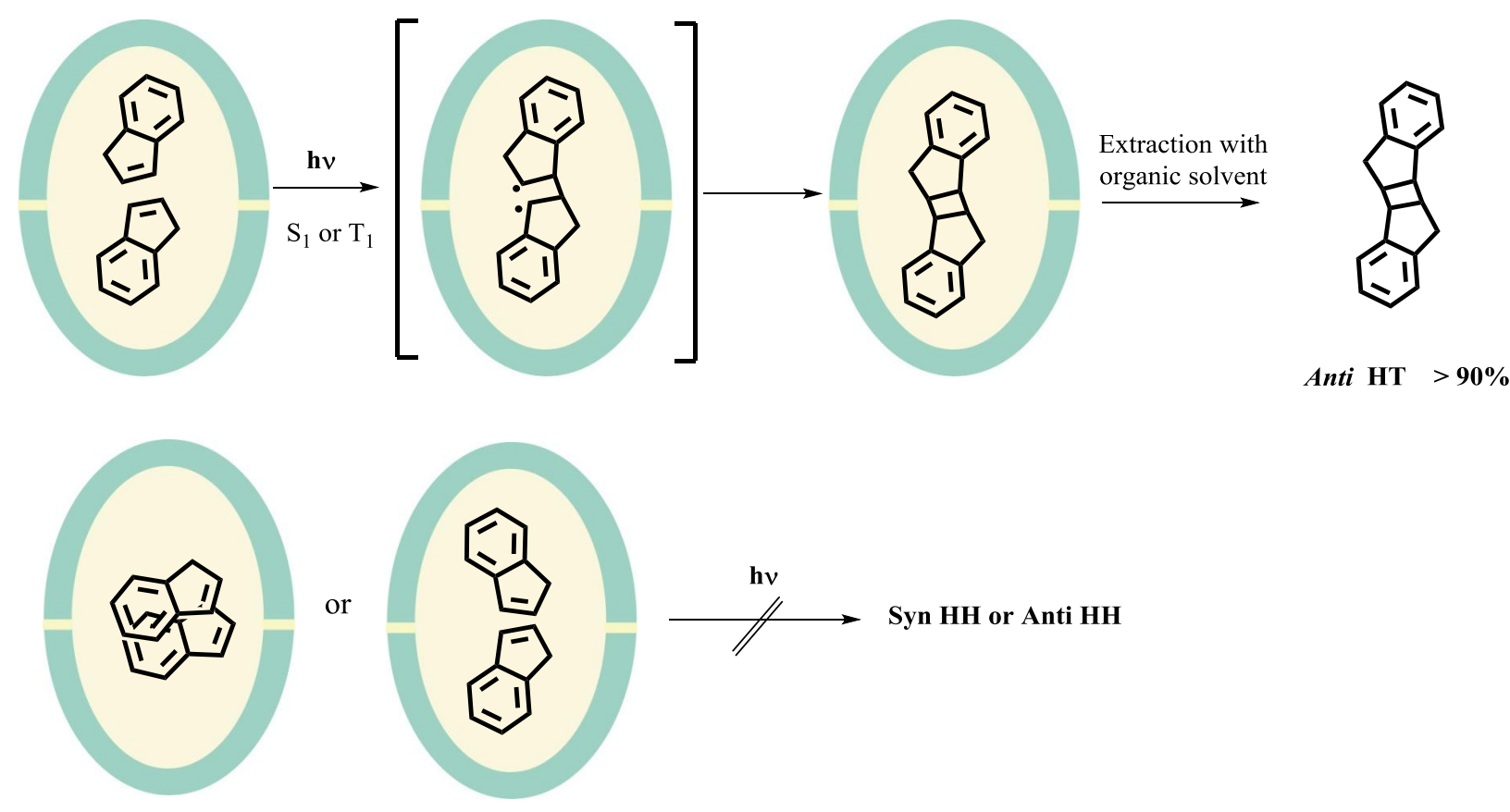

Figure 7. Proposed reaction pathway (After the photoreaction, the organic solvent soluble products were extracted from $\mathrm{OA}$ capsule with $\mathrm{CDCl}_{3}$, and analyzed to calculate product distribution).

The photodimerization is carried out in a pyrex tube (> $290 \mathrm{~nm}$ ) where OA absorbs light predominantly.[22, 29] Hence, we believe the reaction takes place by energy transfer from host to encapsulated guest (indene) that would result in formation of excited triplet of indene. The excited triplet reacts further to yield the final photodimer, anti-head-tail, through a radical 
intermediate. The proposed mechanism is shown in Figure 7. The studies suggest the product control exhibited by OA capsule is primarily due to pre-organization of the starting material; the other controlling factor could be the dimension of the product formed, i.e., better fit of the antihead-tail dimer over the other dimeric products. Moreover, indene is known to undergo light catalyzed polymerization in solvents like benzene. Encapsulation of guest(s) with OA not only allows one to carry out reactions in aqueous medium and in benzene like environment but also limits the number of molecules reacting thereby imposing a 'controlled reactivity' on included substrates; polymerization is prohibited.

\section{Conclusion}

It is known that the hydrophobic cavity of water soluble cavitand OA could help dissolve a variety of organic, otherwise water insoluble guest molecules in the aqueous medium. The above results with indene, in conjunction with other studies from our labs, clearly show that OA interior, besides solubilizing, preorganizes the guest molecules to yield a dimer that is not generally formed under conventional conditions. The high selectivity observed can be attributed to the weak, non-covalent interaction and the available free space within the OA capsule due to guest occupancy. In addition, it appears that the photodimer that has a snug fit within the OA capsule is favored. We envision that knowledge gained by studying photoreactions in the cavitand based supramolecular systems will have their utility in organic synthesis for effecting chemical transformations that are otherwise challenging in isotropic media.

\section{Acknowledgements}

VR is grateful to the National Science Foundation for financial support 


\section{References}

[1] N.J. Turro, V. Ramamurthy, J.C. Scaiano, Modern Molecular Photochemistry of Organic Molecules, University Science Books, Sausalito, CA, 2010.

[2] R.G. Weiss, V. Ramamurthy, G.S. Hammond, Photochemistry in Organized and Confining Media: A Model, Acc. Chem. Res., 26 (1993) 530-536.

[3] G.M.J. Schmidt, Photodimerization in the solid state, Pure Appl. Chem, 27 (1971) 647-678.

[4] V. Ramamurthy, K. Venkatesan, Photochemical reactions of organic crystals, Chem. Rev., 87 (1987) 433-481.

[5] V. Ramamurthy, R.G. Weiss, G.S. Hammond, A Model for the Influence of Organized Media on Photochemical Reactions, in: D.H. Volman, G.S. Hammond, D.C. Neckers (Eds.) Advances in Photochemistry, 1993.

[6] V. Ramamurthy, Photochemistry in Organized \& Constrained Media, in, VCH, New York, 1991.

[7] K. Muthuramu, N. Ramnath, V. Ramamurthy, Photodimerization of coumarins in micelles: limitations of alignment effect, J. Org. Chem., 48 (1983) 1872-1876.

[8] K. Muthuramu, V. Ramamurthy, Photodimerization of Coumarin in Aqueous and Micellar Media, J. Org. Chem., 47 (1982) 3976-3979.

[9] J.W. Lee, S. Samal, N. Selvapalam, H.-J. Kim, K. Kim, Cucurbituril Homologues and Derivatives: New Opportunities in Supramolecular Chemistry, Acc. Chem. Res., 36 (2003) 621630. 
[10] L.S. Kaanumalle, R. Ramesh, V.S.N.M. Maddipatla, J. Nithyanandhan, N. Jayaraman, V. Ramamurthy, Dendrimers as Photochemical Reaction Media. Photochemical Behavior of Unimolecular and Bimolecular Reactions in Water-Soluble Dendrimers, J. Org. Chem, 70 (2005) 5062-5069.

[11] C.L.D. Gibb, B.C. Gibb, Well-Defined, Organic Nanoenvironment in Water: The Hydrophobic Effect Drives a Capsular Assembly, J. Am. Chem. Soc., 126 (2004) 11408-11409. [12] V.T. D'Souza, M.L. Bender, Miniature organic models of enzymes, Acc. Chem. Res., 20 (1987) 146-152.

[13] R. Breslow, Artificial Enzymes, in, Wiley-VCH, Weinheim, 2005.

[14] R. Breslow, S.D. Dong, Biomimetic Reactions Catalyzed by Cyclodextrins and Their Derivatives, Chem. Rev., 98 (1998) 1997-2011.

[15] S. Arumugam, D.R. Vutukuri, S. Thayumanavan, V. Ramamurthy, A styrene based water soluble polymer as a reaction medium for photodimerization of aromatic hydrocarbons in water, J. Photochem. Photobiol., A, 185 (2007) 168-171.

[16] S. Karthikeyan, V. Ramamurthy, Templating Photodimerization of Coumarins within a Water-Soluble Nano Reaction Vessel, J. Org. Chem., 71 (2006) 6409-6413.

[17] R. Kaliappan, M.V.S.N. Maddipatla, L.S. Kaanumalle, V. Ramamurthy, Crystal engineering principles applied to solution photochemistry: controlling the photodimerization of stilbazolium salts within gamma -cyclodextrin and cucurbit[8]uril in water, Photochem. Photobiol. Sci. , 6 (2007) 737-740.

[18] V. Ramamurthy, S. Gupta, Supramolecular photochemistry: from molecular crystals to water-soluble capsules, Chem. Soc. Rev., 44 (2015) 119-135. 
[19] L.S. Kaanumalle, C.L.D. Gibb, B.C. Gibb, V. Ramamurthy, A Hydrophobic Nanocapsule Controls the Photophysics of Aromatic Molecules by Suppressing Their Favored Solution Pathways, J. Am. Chem. Soc., 127 (2005) 3674-3675.

[20] M. Porel, N. Jayaraj, L.S. Kaanumalle, M.V.S.N. Maddipatla, A. Parthasarathy, V. Ramamurthy, Cavitand Octa Acid Forms a Nonpolar Capsuleplex Dependent on the Molecular Size and Hydrophobicity of the Guest, Langmuir 25 (2009) 3473-3481.

[21] L.S. Kaanumalle, V. Ramamurthy, Photodimerization of acenaphthylene within a nanocapsule: excited state lifetime dependent dimer selectivity, Chem. Commun., (2007) 10621064.

[22] A. Parthasarathy, L.S. Kaanumalle, V. Ramamurthy, Controlling Photochemical Geometric Isomerization of a Stilbene and Dimerization of a Styrene Using a Confined Reaction Cavity in Water, Org. Lett. , 9 (2007) 5059-5062.

[23] A. Parthasarathy, S.R. Samanta, V. Ramamurthy, Photodimerization of hydrophobic guests within a water-soluble nanocapsule, Res Chem Intermed, 39 (2013) 73-87.

[24] M. Yasuda, C. Pac, H. Sakurai, Photochemical reactions of aromatic compounds. XXXIII. Photoreactions of 1-cyanonaphthalene with indene in various solvents, Bull. Chem. Soc. Jpn., 53 (1980) 502-507.

[25] W. Metzner, D. Wendisch, Photodimerization of indene, Justus Liebigs Ann. Chem., 730 (1969) 111-120.

[26] C. DeBoer, Inefficiency in the photosensitized dimerization of indene, J. Am. Chem. Soc., 91 (1969) 1855-1856.

[27] T. Majima, C. Pac, A. Nakasone, H. Sakurai, Redox-photosensitized reactions. 7. Aromatic hydrocarbon-photosensitized electron-transfer reactions of furan, methylated furans, 1,1- 
diphenylethylene, and indene with p-dicyanobenzene, J. Am. Chem. Soc., 103 (1981) 44994508.

[28] L. Brancaleon, D. Brousmiche, V.J. Rao, L.J. Johnston, V. Ramamurthy, Photoinduced Electron Transfer Reactions within Zeolites: Detection of Radical Cations and Dimerization of Arylalkenes, J. Am. Chem. Soc., 120 (1998) 4926-4933.

[29] P. Jagadesan, B. Mondal, A. Parthasarathy, V. Jayathirtha Rao, V. Ramamurthy, Photochemical reaction containers as energy and electron-transfer agents, Org, Lett., 15 (2013) 1326-1329. 
TOC
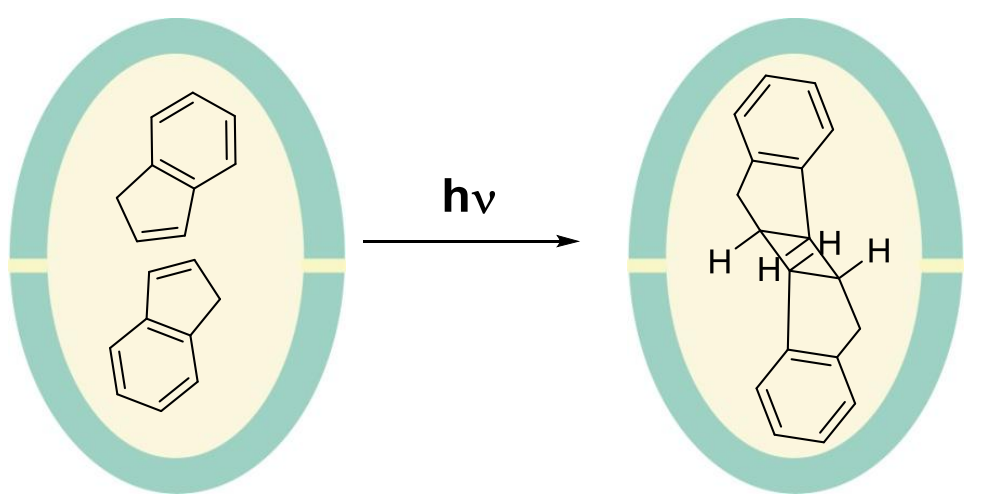

anti head-tail dimer 